\title{
Dissociation of stress and food-deprivation effects on spatial performance
}

\author{
KATHERINE A. MILLER and NANCY K. DESS \\ Occidental College, Los Angeles, Califormia
}

\begin{abstract}
Food deprivation and exposure to a stressor both influence spatial performance. These effects may not be independent, because stress changes eating and body weight. The present study dissociated stress from eating and body weight to determine whether stress alters spatial performance indirectly via ingestive processes. Some rats experienced inescapable tailshock accompanied by reduced eating and body weight (STRESS+DEP), while others experienced only stress (STRESS) or only reduced eating and body weight (DEP). Eight-arm radial maze exploration was assessed in all groups. Exploration quality was similar across groups, and amount of exploration differed most between the groups that differed least with respect to deprivation: DEP rats explored more than did STRESS+DEP rats. Thus, stress apparently does not alter exploration by virtue of a change in ingestion. However, stressed rats that were spared reduced caloric intake and body weight (STRESS) explored more than did STRESS+DEP rats, suggesting that food can attenuate stress-induced reductions in exploration.
\end{abstract}

Exploration benefits opportunistic feeders, such as rats, by allowing mapping of the environment with respect to feeding opportunities or the lack thereof. Traversal of a radial-arm maze models natural foraging and allows measurement of several aspects of spatial exploration. Quantitative features include the total number of arms entered and traversed and how quickly. Qualitative features include the efficiency and patterning of arm choices. Exploring efficiently consists of selecting novel versus familiar arms and making small-angle transitions between arms (Dale \& Roberts, 1986; FitzGerald, Isler, Rosenberg, Oettinger, \& Battig, 1985; Olton \& Samuelson, 1976; Schenk, Contant, \& Grobety, 1990; Sherrick, Brunner, Roth, \& Dember, 1979; Timberlake, 1983).

Food deprivation is one of many modulators of spatial exploration. Food-deprived rats run more (Routtenberg \& Kuznesof, 1967) and are more efficient in exploring a maze than are nondeprived rats (Dale \& Roberts, 1986; Timberlake \& White, 1990). Interestingly, maze exploration by hungry rats tends to be nonredundant even when no food is found (FitzGerald et al., 1985, Experiment 2). In fact, information about where food cannot be found, rather than where food can be found, may be more influential in arm choices: rats seem to act primarily to avoid nonreward (Olton, 1972; Olton, Walker, Gage, \& Johnson, 1977).

Stress also affects spatial performance. Shors and Dryver (1992) reported that exposure to intermittent inescapable tailshock increased the number of trials rats required before visiting the arms of a baited radial-arm maze nonre-

This work was supported by a research grant from the Ford Foundation administered by Occidental College and awarded to K.A.M. We thank Steve Chang for assistance in data collection. Correspondence concerning this article should be addressed to N. K. Dess, Department of Psychology, Occidental College, 1600 Campus Road, Los Angeles, CA90041 (e-mail: dessnk@oxy.edu). dundantly. However, stress also delayed initial maze exploration. When the data were adjusted for initial delay of exploration, the apparent effect of stress on learning rate diminished. Thus, stress affected how much and how soon the rats explored more than it did their ability to learn to find food efficiently.

Stress also can enhance spatial performance. In a water escape task, mild stress facilitates navigation by salient, relevant cues (Lee \& Maier, 1988) and attenuates the deficit otherwise seen in rats housed in isolation (Wade \& Maier, 1986). These studies differed from Shors and Dryver's (1992) in several respects, for example, in the use of mild stressors and an aversively motivated task, and many factors could have contributed to the disparate effects. One candidate is the relationship between stress and ingestion. Stress affects eating and body weight (e.g., Dess, Minor, \& Brewer, 1989; Dess, Raizer, Chapman, \& Garcia, 1988; Paré, 1965; Weiss, 1968). While stress could directly influence the cognitive or emotional bases of spatial performance, it could also act indirectly by modulating ingestive processes. Stress usually decreases food intake, but an increase occurs under some conditions (e.g., Bertiere, Mame Sy, Baigts, Mandenoff, \& Apfelbaum, 1984). Thus, by influencing ingestion, stress could either impair or enhance spatial performance. This interpretation is consistent with the regulatory shift hypothesis, according to which energy regulation is a final common path for stress and a range of other environmental and intraorganismic events (Dess, 1991).

The present study was conducted to dissociate the effects of stress from those of changes in eating and body weight on exploration of a radial-arm maze. One group of rats (STRESS+DEP) was exposed to a session of inescapable tailshock followed by daily mild stressor reinstatement; food intake and body weight were expected to drop (Dess et al., 1989). A second group (DEP) was not stressed, but food was rationed to reduce calorie intake and body weight. 
The third group (STRESS) was stressed and received a highcalorie diet supplement to keep eating and body weight at or above preexperimental levels (Dess \& Choe, 1994; Vaswani, Tejwani, \& Mousa, 1983). If stress affects spatial performance by altering ingestive processes, then reducedeating groups (STRESS + DEP, DEP) should behave similarly to each other and differently from a stressed-but-replete group (STRESS). If, on the other hand, stress affects ingestion and spatial performance through separate mechanisms, then stressed groups (STRESS+DEP, STRESS) should behave similarly to each other and differently from a nonstressed group (DEP).

\section{METHOD}

\section{Subjects}

Twenty-four male Holtzman rats $(315-355 \mathrm{~g})$ from HSD Inc. (Indianapolis, IN) were used. At least 1 week prior to the start of the experiment, they were housed individually on a 12:12 light:dark schedule with light onset at 7:30 p.m. PST. Treatments and measurements took place in the dark phase, between 9:00 a.m. and 2:30 p.m., in red light. Purina 5001 Chow was available in the home cages prior to and during the experiment, though on a limited basis for the DEP group during testing. Tap water was available in the home cages at all times.

\section{Apparatus}

The initial stress session was administered in individual acrylic boxes $(14 \times 11 \times 17 \mathrm{~cm})$. A rat's tail protruded through the end of the box and was taped to an acrylic rod; two electrodes coated with electrode paste were taped to the tail $1.5 \mathrm{~cm}$ apart. On test days, reinstating shock was administered in an acrylic box $(32 \times 18 \times 20 \mathrm{~cm}$, $\mathrm{L} \times \mathrm{W} \times \mathrm{H}$ ) lined by two steel plates that angled inward from the top of each side of the box and flattened to form a platform $5 \mathrm{~cm}$ wide with a $1.5-\mathrm{cm}$ gap between the plates. When placed in the box, a rat straddled the gap, with a front and a rear foot on each plate to complete a circuit. In both the original and reinstating stress sessions, shock was delivered from a constant-current shock generator (Lafayette Instruments Model 82400, Lafayette, IN) to boxes located in individual white sound-attenuating chambers. Each chamber was illuminated by a bulb in the center of the front wall, and ventilation fans and a white-noise generator provided $80-\mathrm{dB}$ masking noise.

The radial-arm maze stood $102 \mathrm{~cm}$ high and consisted of a 20.5 $\mathrm{cm}$-diam platform with eight arms radiating at $45^{\circ}$ angles to each other. Each arm, $81 \mathrm{~cm}$ long and $8 \mathrm{~cm}$ wide, was enclosed by sidewalls. The right sidewall was $3 \mathrm{~cm}$ high; the first $20 \mathrm{~cm}$ of the left sidewall was $11 \mathrm{~cm}$ high and the rest was $3 \mathrm{~cm}$ high. The alleys of the arms were alternately painted gray and black. The platform was black and the sidewalls were gray. The maze was located in a room featuring red light and various landmarks (a door, wall posters, experimenter on a stool).

To hold food reinforcement constant across groups, arm entries, and trials, the maze was unbaited. An unbaited maze also was appropriate for other reasons. Shors and Dryver (1992), who used a baited maze, concluded that stress primarily affected initiation of exploratory activity, not learning to find food efficiently. If this analysis is correct, the effect of stress should be replicable in an unbaited maze. Moreover, maze exploration is influenced by deprivation regardless of food reward (FitzGerald et al., 1985; Timberlake \& White, 1990), and the absence of food is at least as important to maze performance as is its presence (Olton, 1972; Olton et al., 1977).

\section{Procedure}

Baseline food intake and body weight were assessed for $48 \mathrm{~h}$. Rats then were assigned to three groups of 8 rats each, matched for body weight and food intake: stress plus accompanying food deprivation and weight loss (STRESS +DEP), deprivation only (DEP), and stress only (STRESS). STRESS +DEP and STRESS animals were exposed to 60 0.6-mA, 5-sec shocks on a 60-sec variable time (VT) schedule (range 20-120 sec), beginning at about 9:00 a.m. DEP rats remained in their home cages; through restriction of their food allowances, daily changes in the DEP rats' body weights were roughly yoked to those of the STRESS + DEP group. Yoking could be only approximate because stress-induced weight loss results in part from decreased gut efficiency and lipolysis, processes we could not directly control (Dess et al., 1989, Experiment 3; Rivest, Deshaies, \& Kostowski, 1989).

After stress and throughout testing, rats in the STRESs group were given unrestricted access to a 2:1 (weight/weight) mash of Keebler Pecan Sandies cookies in Carnation sweetened condensed milk, in addition to chow and water. Inescapable tailshock increases consumption of easily eaten and absorbed nutrients such as sucrose (Dess, 1992; Dess \& Choe, 1994), so the STREss rats were expected to maintain or exceed baseline caloric intake and body weight.

Maze testing occurred between 9:15 a.m. and 2:30 p.m. on 4 consecutive days, beginning the day after the stress session. Before the first maze trial on each day, the shocked groups (STRESS + DEP, STRESS) were given five $0.6-\mathrm{mA}, 5-\mathrm{sec}$ reinstating footshocks on a 60 -sec VT schedule (range $20-120 \mathrm{sec}$ ). They remained in the box for $15 \mathrm{~min}$ after the last shock and then were placed in the maze. For all rats, the second trial began at approximately $12: 30$ p.m.

To begin a trial, a rat was placed in the center of the maze facing in a quasi-random orientation. It then was allowed to move through the maze until it had made eight choices or until $10 \mathrm{~min}$ had elapsed, whichever came first. The experimenter recorded each arm entry, whether the arm was completely traversed, and the time taken to enter eight arms. A rat "entered" an arm when all four feet were in the arm simultaneously and "fully traversed" the arm when its nose reached within $2 \mathrm{~cm}$ of the end of the arm. Nonredundant arm entries and adjacent arm entries, a measure of small-angle transitioning, were calculated later.

The maze was thoroughly cleansed with dilute Alconox detergent between trials. The order in which groups and rats within a group were run on the maze was balanced over trials.

Throughout the experiment, food intake was measured daily before reinstatement began. Body weight was measured daily between the two maze trials.

\section{RESULTS}

Data on each dependent measure were analyzed with analyses of variance (ANOVA), accompanied by contrasts using an appropriate error term. All test statistics significant at $\alpha=.05$ are reported in the text.

\section{Food Intake and Body Weight}

Figure 1 shows caloric intake (upper panel) and weight gain (lower panel) during maze testing. Caloric intake in the STRESS condition was well above baseline levels; STRESS rats ate about $90 \%$ of their calories as cookie mash throughout testing (not shown). Caloric intake in the STRESS+DEP and DEP groups remained below baseline throughout testing. However, DEP rats' caloric intake rose over days as food rations were increased to yield weight gain closer to that of the STRESS+DEP group.

These trends in caloric intake were reflected in body weight. STRESS rats were at or above baseline throughout testing, whereas the STRESS+DEP and DEP groups remained below baseline. STRESS and DEP rats gained weight during testing, but STRESS+DEP rats did not.

Mixed design ANOVAs with group and test day as variables yielded a main effect of group for both caloric intake 


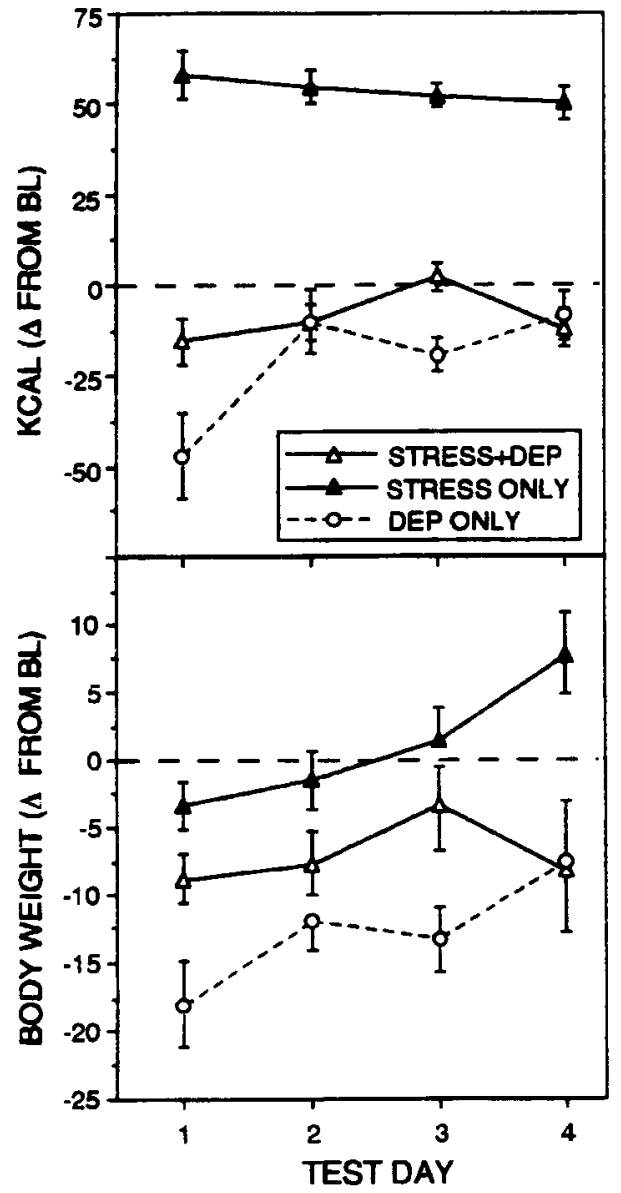

Figure 1. Caloric intake (upper panel) and body weight (lower panel) in STRESS+DEP, STRESS, and DEP groups on each day of maze testing, as a change from baseline.

and weight change $[F \mathrm{~s}(2,21)=67.90$ and 7.62 , respectively]. Contrasts revealed that the two restricted-food groups (STRESS+DEP, DEP) did not differ on either dependent measure but, taken together, differed from the STRESS group on caloric intake and weight change $[t \mathrm{~s}(21)=11.53$ and 3.57 , respectively]. The group $\times$ day interaction also was significant for caloric intake and weight change $\left[F_{\mathrm{s}}(6,63)=6.19\right.$ and 2.88 , respectively $]$. Caloric intake increased significantly from Day 1 to Day 4 only in the DEP group $[t(84)=5.80]$. Weight gain from the beginning to the end of testing was significant in the STRESS and DEP groups $[t \mathrm{~s}(84)=4.38$ and 4.04 , respectively], but not in the STRESS+DEP group.

\section{Maze Exploration}

Maze exploration was examined first in terms of quantity. Stress reduced exploration, particularly if accompanied by a reduction in food intake and body weight. Armentry rate (upper panel, Figure 2) was calculated for each trial by dividing the number of arm entries by time on the maze; rats that failed to make eight entries were assigned the maximum maze time of $10 \mathrm{~min}$. Exploration by STRESS+DEP rats was slower than that of DEP rats throughout testing, and the STRESS group was intermediate. A mixed design ANOVA with group and test day as variables yielded a main effect of group $[F(2,21)=5.25]$. Contrasts showed that DEP explored significantly faster than did STRESS+DEP $[t(21)=3.24]$ but only marginally significantly faster than did STRESS $[t(21)=1.93, p<.10]$. The two stressed groups did not differ from each other.

Full arm traversal was analyzed as percentage of total arm entries. Some rats' relative inactivity posed two problems. First, percentages based on a few values can be unduly influenced by a single observation. For instance, one full traversal in three entries yields a low rate $(33 \%)$, whereas a single additional full traversal $(2 / 3$, or $66 \%)$ makes full traversal appear common. To reduce this problem, trials with fewer than four arm entries were excluded; if both trials on a test day failed to meet the criterion, the rat was excluded from that day's analysis. On this basis, 1 STRESS+DEP rat was excluded on Day $1(n=7)$ and 2 STRESS+DEP rats were excluded on Days 2-4 $(n=6)$. Second, the usual deletion

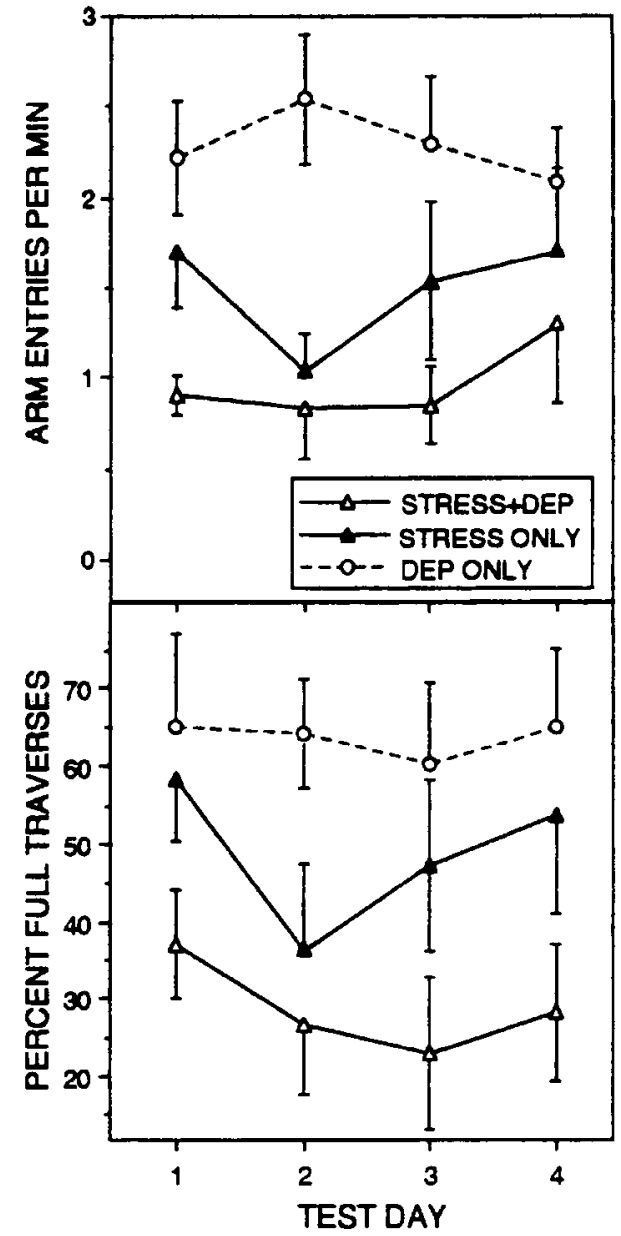

Figure 2. Rate of arm entries per minute (upper panel) and percentage of arm entries culminating in full traversal (lower panel) averaged over two trials on each test day. (See text for exclusion criteria for full traversal measure.) 
of incomplete cases from repeated measures ANOVAs increases the risk of selective attrition and reduces statistical power. These problems were minimized by using a separate between-group ANOVA on each test day: Each day's ANOVA would include all rats meeting inclusion criteria on that day, some of whom would have been excluded altogether from a repeated measures ANOVA because of insufficient activity on some other day. A mixed design (group $\times$ day) ANOVA on data from complete cases was used to assess any changes over test days.

Percentage of full traversals is shown in the lower panel of Figure 2. Overall, STRESS + DEP rats fully traversed a smaller percentage of entered arms than did the DEP group; the STRESS group was intermediate. The main effect of group was significant on Day $2[F(2,19)=4.24]$, on which STRESS+DEP and STRESS differed from DEP $[t(19)=2.71$ and 2.18 , respectively $]$. Though the group main effect was only marginally significant on Days 3 and $4[F(2,19)=2.90$ and $2.65, p s<.10]$, contrasts showed that STRESS+DEP differed from DEP on these days as well $[t(19)=2.41$ and 2.27 , respectively $]$. The STRESS group did not differ significantly from either of the other groups after Day 2. It is important to note that these differences occurred despite the elimination of the least active STRESS+DEP rats from the analyses. A mixed design ANOVA with group and test day as variables yielded no day effects.

Measures of exploration quality included percentage of nonredundant arm entries and percentage of adjacent arm entries. These measures were analyzed in the same manner as percentage of full traversals, with one necessary change. Minimum number of arm entries was increased from four to five, because "nonredundant" and "adjacent" are undefined until the second arm choice. Using these criteria, 3 STRESS+DEP rats were excluded on Day 2 for nonredundant entries and on Days 2 and 4 for adjacent arms $(n=5)$; on all other days, 2 STRESS+DEP rats were excluded on each measure $(n=6)$.

Percentage of nonredundant and adjacent arm entries are shown in Figure 3. These measures of exploration efficiency show no effect of stress, whether accompanied by decreased or by increased ingestion. Between-group ANOVAs on each test day yielded no significant group effects. Mixed design ANOVAs with group and day as variables yielded no day effects for either measure.

\section{DISCUSSION}

This study used conditions of stress plus deprivation, stress only, and deprivation only to dissociate the effect of stress per se from indirect effects of stress-induced changes in ingestive processes. If stress affects exploration indirectly via ingestion, then the groups' order on exploratory measures should have matched their order in terms of eating and body weight. It did not. Despite group differences in caloric intake and weight, no differences were apparent in exploration quality. With respect to the quantity of exploration, the groups with similarly reduced ingestion behaved differently from each other, and the stress-only rats' exploration was generally intermediate despite higher

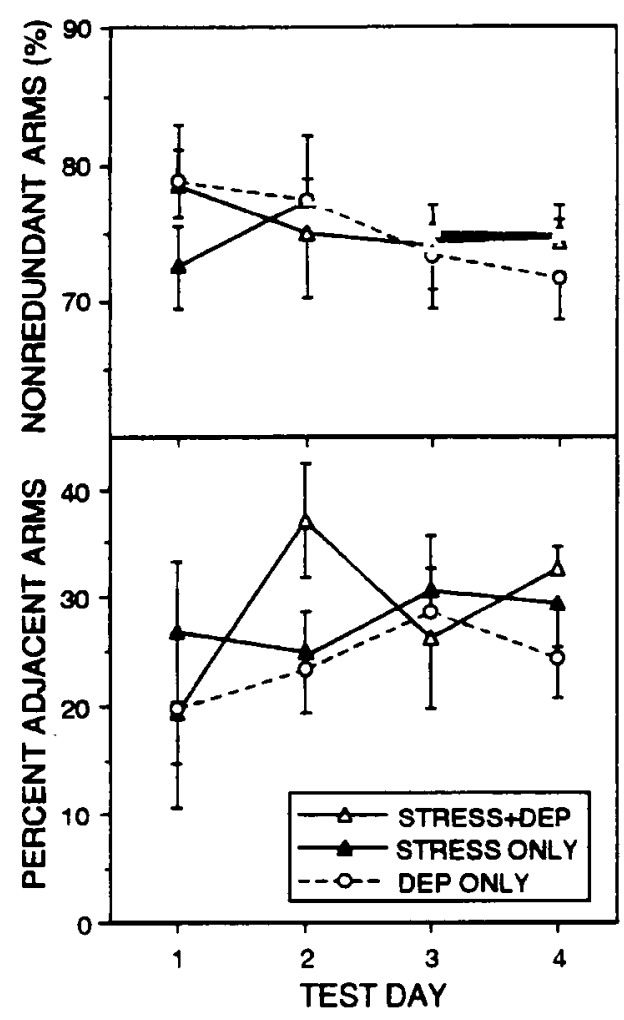

Figure 3. Percentage of arm entries that were nonredundant with entries earlier in the same trial (upper panel) and percentage of entries to arms adjacent to preceding arm entry (lower panel) averaged over two trials on each test day. (See text for exclusion criteria.)

caloric intake. This pattern suggests that stress and food deprivation do not influence exploration through a final common path.

The impairment of spatial performance by stress in the present study concurs with the impairment observed by Shors and Dryver (1992) - including the effect of stress primarily on exploration quantity - and contrasts with the facilitation observed by others (Lee \& Maier, 1988; Wade $\&$ Maier, 1986). Such concurrence might have been expected on the basis of the similarity of the stressors and spatial tasks used. The results are not identical, though: Shors and Dryver (1992) reported more initial perseveration after stress and changes in performance over days, neither of which was apparent here. The present procedures did not, however, directly replicate Shors and Dryver's, which included more severe stress, a baited maze, maze preexposure, and calculation of an "acquisition index" more appropriate to a food-rewarded task. These procedural differences could easily account for differences in outcomes. The important point is that the suppression of exploration by stress was observed in both studies, which speaks to the robustness of this phenomenon.

The present study also clarifies the nature of the phenomenon. It is unlikely that the stressed rats in Shors and Dryver (1992) ate more than did nonstressed controls and therefore explored less (Timberlake \& White, 1990). Con- 
ceivably, however, reduced eating by the stressed group could have increased their exploration, leading to underestimation of the stressor's impact. As little information was given about food intake and body weight, their role in the outcome could not be evaluated. The experimental control of ingestion in the present study provided an explicit test of whether stress-induced changes in ingestion played an important role in stressed rats' exploration, yielding the answer "no." Moreover, the results of these two studies are similar despite the use of an unbaited maze in the present study. Differential responding to reward or nonreward probably was not responsible for the stress effect in either study.

Imperfect yoking of Groups STRESS+DEP and DEP potentially compromises these conclusions. Initial weight loss was somewhat less among STRESS + DEP rats than among DEP rats, perhaps accounting for their decreased exploration. However, the caloric intake and body weight of the two groups converged over days with no corresponding convergence in exploration. Moreover, if a smaller ingestive deficit were responsible for less exploration by STRESS+DEP rats, then "overfeeding" stressed rats should have exaggerated the difference. To the contrary, the overfed stressed group (STRESS) explored more than did Group STRESS+DEP. Thus, imperfect yoking cannot account for the group differences in exploration.

The behavior of the STRESS-only group implies that stress is not sufficient for reduced exploration. Though exposed to the same stressor, rats given supplemental feeding (STRESS) explored somewhat more than did STRESS+DEP rats. Stress-induced food deprivation apparently does not cause reduced exploration, but boosting ingestion after stress with calorically dense foods can attenuate the effect.

Feeding to repletion generally reduces exploration (Timberlake \& White, 1990), whereas overfeeding stressed rats increased exploration in the present study. Thus, stress and reduced eating interact to modulate exploration. The absence of a nonstressed, nondeprived group poses no threat to this conclusion. Nondeprived rats are less active in a maze than are food-deprived rats (e.g., Dale \& Roberts, 1986; Timberlake \& White, 1990). Assuming that such a group would have explored less than Group DEP, the effects of deprivation and stress still would have been nonadditive.

The present findings provide partial support for the regulatory shift hypothesis (Dess, 1991), according to which some effects of stress comprise an energy conservation strategy in the face of risky feeding opportunities. This approach can accommodate the hesitance of stressed rats to explore new areas and objects (Katz, Roth, \& Carroll, 1980; Rosellini \& Widman, 1989; Shors \& Dryver, 1992). In the present study, a role for energy regulation is supported by greater exploration by stressed rats given milk and cookies, who can afford to be less energetically conservative. High caloric intake may disarm the mechanisms that cause a stressed animal to suspend exploration and utilize stored fuels. But, while reduced exploration after stress is consistent with the regulatory shift hypothesis, findings originally cited in support of the hypothesis in- cluded parallels between stress and experimentally imposed food deprivation. With respect to exploration, the present results show that stress cannot be modeled with extrinsic food deprivation. This stress effect is not mediated by central or peripheral processes directly controlled by food availability.

The suppression of exploration by stress and its alleviation by supplemental feeding may occur via emotional states such as depression or anxiety (Katz et al., 1980; Shors \& Dryver, 1992). Central mechanisms of energy regulation may generate these emotions (Minor, 1994), which then mediate behavioral coping strategies such as place neophobia (Minor, Dess, Ben David, \& Chang, 1994) and increased sugar and fat intake (Dess, 1992; Dess \& Choe, 1994). There is good evidence that the latter phenomena occur by virtue of stress-reducing properties of sugar and fat (Blass, Shide, \& Weller, 1989). Given that eating carbohydrates can induce lethargy (Christensen \& Burrows, 1990; Kushner \& Mook, 1984; Wurtman \& Wurtman, 1989), the increased exploration by STRESS rats in this study cannot be the direct consequence of eating milk and cookies. The effect is more likely caused by the impact of supplemental feeding on emotional processes.

In the present study, stress accompanied by food deprivation and weight loss decreased exploration. Though this particular effect apparently was not a byproduct of ingestion, controlling for ingestive changes in studies of other stress effects may sometimes prove worthwhile. The yoking and supplemental feeding procedures employed here are a means of doing so.

\section{REFERENCES}

Bertiere, M. C., Mame Sy, F., Baigts, F., Mandenoff, A., \& ApfelBAUM, M. (1984). Stress and sucrose hyperphagia: Role of endogenous opiates. Pharmacology, Biochemistry \& Behavior, 20, 675-679.

Blass, E. M., Shide, D. J., \& Weller, A. (1989). Stress-reducing effects of ingesting milk, sugar, and fats: A developmental perspective. In L. H. Schneider, S. J. Cooper, \& K. A. Halmi (Eds.), The psychobiology of human eating disorders: Preclinical and clinical perspectives (Annals of the New York Academy of Sciences, Vol. 575, pp. 292-306). New York: New York Academy of Sciences.

Christensen, L., \& Burrows, R. (1990). Dietary treatment of depression. Behavior Therapy, 21, 183-193.

DALE, R. H., \& RoBerTS, W. A. (1986). Variations in radial maze performance under different levels of food and water deprivation. Animal Learning \& Behavior, 14, 60-64.

DESs, N. K. (1991). Ingestion and emotional health. Human Nature, 2 , 235-269.

Dess, N. K. (1992). Divergent responses to saccharin and sucrose availability after stress in rats. Physiology \& Behavior, 52, 115-125.

Dess, N. K., \& ChOE, S. (1994). Stress selectively reduces sugar+saccharin mixture intake but increases proportion of calories consumed as sugar by rats. Psychobiology, 22, 77-84.

DESS, N. K., MinOR, T. R., \& BREWER, J. (1989). Suppression of feeding and body weight by inescapable shock: Modulation by quinine adulteration, stress reinstatement, and controllability. Physiology \& Behavior, 45, 975-983.

Dess, N., Raizer, J., Chapman, D., \& Garcia, J. (1988). Stressors in the learned helplessness paradigm: Effects on body weight and conditioned taste aversion in rats. Physiology \& Behavior, 44, 483-490.

FitzGerald, R. E., Isler, R., Rosenberg, E., Oettinger, R., \& BatTIG, K. (1985). Maze patrolling by rats with and without food reward. Animal Learning \& Behavior, 13, 451-462. 
Katz, R. J., Roth, K. A., \& Carroll, B. J. (1980). Acute and chronic stress effects on open field activity in the rat: Implications for a model of depression. Neuroscience \& Biobehavioral Reviews, 5, 247-251.

Kushner, L. R., \& Mook, D. G. (1984). Behavioral correlates of oral and postingestive satiety in the rat. Physiology \& Behavior, 33, 713 . 718.

LEE, R. K. K., \& MAIER, S. F. (1988). Inescapable shock and attention to internal versus external cues in a water discrimination escape task. Journal of Experimental Psychology: Animal Behavior Processes, 14 302-310.

MinoR, T. R. (1994, November). When fear is too much: Neuronal exhaustion and adenosine-mediated conservation withdrawal. Paper presented at the annual meeting of the Psychonomic Society, St. Louis.

Minor, T. R., Dess, N. K., Ben David, E., \& Chang, S. (1994). Individual differences in vulnerability to inescapable shock in rats. Journal of Experimental Psychology: Animal Behavior Processes, 20, 402-412.

OLTON, D. S. (1972). Discrimination behavior in the rat: Differential effects of reinforcement and nonreinforcement. Journal of Comparative \& Physiological Psychology, 79, 284-290.

Olton, D. S., \& SAMUELSON, R. J. (1976). Remembrance of places past: Spatial memory in rats. Journal of Experimental Psychology: Animal Behavior Processes, 2, 97-116.

Olton, D. S., Walker, J. A., Gage, F. H., \& Johnson, G. T. (1977) Choice behavior of rats searching for food. Learning \& Motivation, 8 , 315-331.

PaRé, W. P. (1965). Stress and consummatory behavior in the albino rat Psychological Reports, 16, 399-405.

Rivest, S., Deshaies, Y., \& Kostowski, W. (1989). Effects of corticotropin-releasing factor on energy balance in rats are sex dependent. American Journal of Physiology, 257, R1417-R1422.

Rosellini, R. A., \& WIDMAN, D. R. (1989). Prior exposure to stress reduces the diversity of exploratory behavior of novel objects in the rat (Rattus norvegicus). Journal of Comparative Psychology, 103, 339-346.
RoutTENBERG, A., \& KuZNeSOF, A. (1967). Self-starvation of rats living in activity wheels on a restricted feeding schedule. Journal of Comparative \& Physiological Psychology, 64, 414-421.

Schenk, F., Contant, B., \& Grobety, M.-C. (1990). Angle and directionality affect rat's organization of visit sequences and spatial learning in modular mazes. Learning \& Motivation, 21, 164-189.

Sherrick, M. F., Brunner, R. L., Roth, T. G., \& Dember, W. N. (1979). Rats' sensitivity to their direction of movement and spontaneous alternation behaviour. Quarterly Journal of Experimental Psychology, 31, 83-93.

Shors, T. J., \& Dry ver, E. (1992). Stress impedes exploration and the acquisition of spatial information in the eight-arm radial maze. Psychobiology, 20, 247-253.

TimBerLaKE, W. (1983). Rats' responses to a moving object related to food and water: A behavior-systems analysis. Animal Learning \& Behavior, 11, 309-320.

Timberlake, W., \& White, W. (1990). Winning isn't everything: Rats need only food deprivation and not food reward to efficiently traverse a radial arm maze. Learning \& Motivation, 21, 153-163.

Vaswani, K., Tejwani, G. A., \& Mousa, S. (1983). Stress induced differential intake of various diets and water by rat: The role of the opiate system. Life Sciences, 12, 1983-1996.

WADE, S. E., \& MAIER, S. F. (1986). Effects of individual housing and stressor exposure upon the acquisition of watermaze escape. Learning \& Motivation, 17, 287-310.

WEISS, J. M. (1968). Effects of coping responses on stress. Journal of Comparative \& Physiological Psychology, 65, 251-260.

Wurtman, R., \& Wurtman, J. (1989, January). Carbohydrates and depression. Scientific American, 260, 68-75.

(Manuscript received January 18, 1995; revision accepted for publication October 11,1995 .) 